\title{
Internet-Based Behavioral Interventions for Obesity: An Updated Systematic Review
}

\author{
Gian Mauro Manzoni ${ }^{1,2, *}$, Francesco Pagnini ${ }^{2}$, Stefania $_{\text {Corti }}{ }^{1}$, Enrico Molinari ${ }^{1,3}$ and Gianluca \\ Castelnuovo $^{1,3}$ \\ ${ }^{I}$ Istituto Auxologico Italiano IRCCS, Psychology Research Laboratory, Ospedale San Giuseppe, Verbania, Italy \\ ${ }^{2}$ Department of Psychology, University of Bergamo, Italy \\ ${ }^{3}$ Department of Psychology, Catholic University of Milan, Italy
}

\begin{abstract}
The objective of this systematic review is to update a previous systematic review on the effectiveness of internet-based interventions for weight loss and weight loss maintenance in overweight and obese people with new or additional studies. A literature search from 2008 to March 2010 was conducted. Studies were eligible for inclusion if: participants were adults with a body mass index $\leq 25$, at least one study arm involved an internet-based intervention and the primary aims were weight loss or maintenance. Eight additional studies over the eighteen included in the previous review met the inclusion criteria. Data were extracted on sample characteristics, attrition, weight loss, duration of treatment and maintenance of weight loss. Effect sizes (Hedges g) and relative 95\% confidence intervals were calculated for all two-way comparisons within each study. No attempt was made to pool the data in a meta-analysis because of the great heterogeneity of designs among studies. An examination of effect sizes show that the higher significant effects pertain studies that found a superiority of behavioral internet-based programs enhanced by features such as tailored feedback on selfmonitoring of weight, eating and activity over education only internet-based interventions. However, control groups are very different among studies and this heterogeneity probably accounts for much of the variance in effect sizes. Hence, questions still remain as to the effectiveness of web-based interventions in achieving weight loss or maintenance. Implications for further research include using a "real" control group in order to make meta-analysis possible and developing multi-factorial design in order to separate components of interventions and identify which of them or patterns of them are keys to success.
\end{abstract}

Keywords: Internet, obesity, systematic review, meta-analysis, weight loss, weight loss maintenance.

\section{INTRODUCTION}

In 1997 the World Health Organization (WHO) described obesity as a chronic pathology with such an increasing incidence that it was defined as a global epidemic. Obesity is still an increasingly worrying issue for public health authorities. Indeed, few years ago WHO estimated that by 2015 approximately 2.3 billion adults will be overweight and more than 700 million will be obese [1].

Obesity is associated with early death $[2,3]$ and is universally recognized as a risk factor for many health complications such as cardiovascular diseases, some types of cancer, osteoarthritis, hypertension, dyslipidemia, hypercholesterolemia and diabetes $[1,4]$. These health problems impact heavily on quality of life and involve high treatment costs for the obese people, their families and the public health system. The good news are that overweight and obesity are modifiable

*Address correspondence to this author at the Psychology Research Laboratory, San Giuseppe Hospital, Istituto Auxologico Italiano, Via Cadorna, 90, 28824 Piancavallo (VB), Italy; Tel: 0039-0323-514339;

Fax: 0039-0323-514338; E-mail: gm.manzoni@auxologico.it and that a modest $5-10 \%$ reduction in weight is associated with increased health benefits [5]. The key to success is to achieve an energy balance between calories consumed on one hand, and calories used on the other hand. However, as rightly remarked by Levy et al. [6], both obese people and clinicians should keep in mind that long-term weight loss requires stable changes in behaviors, i.e. eating and exercise habits. This is why behavior therapy is the most researched and best-established method for the treatment of obesity [7]. Indeed, behavior modification is one of five "principles of obesity therapy" along with dietary intervention, physical activity, pharmacotherapy and bariatric surgery [6].

However, clinical practice and research have shown significant difficulties with regard to availability, costs, treatment adherence and long-term efficacy of all the aforementioned procedures $[6,8]$. From a public health strategy point of view, many problems are due to the high costs of these procedures, overall within the recommended continuous care setting [9]. Indeed, the main challenge in the treatment of obesity is to maintain weight loss in the long term [10]. Most overweight and obese individuals regain about one third of the weight lost with treatment within 1 year, sometimes even before the end of the intervention, and they are typically 
back to baseline in 3 to 5 years [11-13]. Furthermore, research has shown that time and travel demands typically associated with traditional weight-loss behavioral programs may contribute to an attrition rate of $20 \%$ in 4-6 months, with greater attrition in longer programs [6, 14]. Given that these interventions are delivered in face-to-face formats, some obese individuals perceive attending treatment sessions as a burden [15] and would prefer to lose weight without having to participate in structured programs involving weekly in-site visits [16].

Therefore, developing effective weight loss programs that are time and cost-saving, appealing and widely accessible has become a health care priority [17]. First research efforts have included the use of telephone [18], interactive television $[19,20]$ and hand-held computers [21, 22]. Then, along with the rapid increase in access to the Internet and the development of numerous commercial programs for weight loss [23, 24], several studies have begun to examine the effectiveness of behavioral weight-loss treatments delivered through the web $[8,25,26]$.

Conceptually, the Internet has the potential to overcome most of limits associated with traditional weight-loss interventions. In addition to being a source for health information accessible 24 hours a day, it offers a number of novel opportunities for self-help programs and also allows healthcare professionals to access and maintain long-term contact with large numbers of overweight or obese individuals in a timesaving and cost-effective manner [8].

Preliminary findings suggest that internet-based interventions may be effective in achieving significant weight loss and weight loss maintenance [25]. However, results are mixed and, as already pointed out by the authors of previous reviews [8, 25, 26], several questions are still unanswered. Hence, no reliable statement can be made at present on the overall effectiveness of internet-based programs in comparison with other kinds of internet-based or more traditional behavioral treatments for weight loss.

The purpose of this review is to update a previous systematic review on the effectiveness of web-based interventions for weight loss and maintenance [25] with new or additional studies.

\section{METHODS}

\section{Study Selection}

All eighteen studies considered in the previous review [25] were directly included also in the present one. An additional literature search restricted to year 2008 up to date was performed using the terms "weight loss", "Internet" and "web-based" in PubMed, PsycINFO, Cochrane Library, The National Institutes of Health Clinical Trials database and the Internet. All identified studies were retrieved and assessed for relevance from the title and abstract by two independent reviewers.

\section{Study Eligibility}

Studies were selected and included in the present review according to 5 criteria: (1) publication in a peer reviewed journal, (2) randomized controlled trial, (3) overweight and/or obese adults as subjects, (4) the Internet as at least one mode of delivery for a weight loss treatment, and (5) weight loss and/or weight loss maintenance as primary outcomes. Apart from 2 of the 18 studies already included in the previous review, a further eight were included in the update after meeting the inclusion criteria.

Of the 8 remaining studies, 5 examined Internet-based weight loss programs either as stand-alone programs or as part of a more comprehensive health intervention, and 3 examined Internet-based weight loss maintenance programs. Studies evaluating weight loss and those evaluating weight loss maintenance were examined separately.

\section{Data Coding}

The full articles were obtained for all studies included in the present review and further inspected to assess their relevance, based on the preplanned criteria for inclusion. Data were independently extracted by three reviewers (GMM, SC and FP) using the following data collection form: (1) number of subjects; (2) kind of the internet-based weight-loss program; (3) kind of the comparison or control condition; (4) subjects typology; (5) mean age; (7) women percentage; (8) length of intervention; (9) recruitment; (10) summary statistics required for computation of effect sizes. The effects of the interventions were assessed through absolute change in body weight.

\section{Calculation of the Effect Sizes and Data Synthesis}

Effect sizes were calculated for all experimental comparisons within each study using Hedges g standardized mean difference. However, effect sizes were not pooled with meta-analysis. We decided not to combine the studies as Neve et al. (2009) made in their review because of two reasons: (1) studies are very heterogeneous in many features of the internet-based programs as well as of the comparison conditions (none of them used a "real" control group); (2) we think that grouping two or three similar studies of the same authors does not improve evidence more that considering them separately. Hence, results were mainly described in a narrative manner. Given that most of studies have been already described in the previous review [25], we considered mainly the new ones.

\section{RESULTS}

\section{Description of Included Studies}

The total number of participants across the studies was 8324 , of which at least $76,7 \%$ were female. Mean age is 46,2 years old. The length of the intervention period ranged from 6 weeks to 2 years. Six interventions were 12 months in duration. Seventeen interventions ranged from 6 weeks to 6 months and two were 2 years. Attrition rates varied from 0 to $70 \%$ at the post-intervention time point. Two studies lost greater than $50 \%$ of participants, eleven lost $20-50 \%$ and twelve $0-20 \%$ with a mean attrition rate of $22,52 \%$. Eighteen interventions recruited participants from community, three interventions through health care system, two studies recruited participants on work sites, one study among university students and one from US Air force.

The additional studies that examined internet-based interventions with the primary aim of achieving weight loss explored four distinct research areas: 1- internet-based programs compared with a control or minimal intervention group ( $\mathrm{n}=4$ ) [27-30]; 2 - generic web-based programs com- 
pared with enhanced web-based programs $(n=1)$ [31]; 3 lifestyle modification program delivered through 5 methods differing for contact frequency and modality (face-to-face, phone or e-mail) $(n=1)$ [32]; 4 - dietary advice plus exercise enhanced by tailored e-mail assistance compared with an exercise-only program $(n=1)$ [33]. The one only additional study with the primary aim of achieving weight loss maintenance following a standard weight loss program compared a control group, which received quarterly newsletters, a group that received face-to-face intervention and a group that received internet-based intervention [11].

\section{Results of Studies Aiming to Achieve Weight Loss}

Effect sizes and relative $95 \%$ confidence intervals for all the studies that examined the effectiveness of internet-based weight loss program are reported in Table $\mathbf{1}$.

The highest effect sizes (negative effect sizes mean a superiority of the first term of the comparison) pertain to Rothert et al.'s study [34] and show that a 6-week individually tailored web-based program was more effective than an information only web-site both at 3 and 6 months. The individually tailored web-based program included individualized educational material and a weight management plan generated automatically from baseline assessments, as well as a buddy system and the ability to e-mail other participants.

As already remarked by Neve et al. (2009), the studies that compared education only web-based interventions with enhanced web-based programs show the highest effect sizes. Apart from Rothert et al. study [34], also Gold et al. [35], Tate et al. $[17,36,37]$ and a more recent study by Pullen et al. [31] obtained medium-high effect sizes comparing an education only web-site with an enhanced web-based program. In particular, Gold et al. reported significantly greater total and percentage weight loss at 6 months and at 12 months from a web-based program with behavioral therapy compared with a commercial web-based program. The behavioral program included the addition of individual e-mail feedback on self-monitoring of weight, eating and activity, as well as homework activities. Tate et al. [37] demonstrated significantly greater weight loss at 3 and 6 months among participants of a web-based program with human e-mail counseling compared with a web-based program alone. There was no significant difference in weight loss achieved by a web-based program with automated counseling when compared with the web-based program alone or the webbased program with human e-mail counseling. The two behavioral interventions included the provision of message boards, a diary to log eating and activity, as well as the feedback on diary entries. However, participants in the human email counseling group received an individualized feedback from a weight loss counselor based on diary entries, while those in the automated group received automated tailored feedback. Tate et al. [36] and Tate et al. [17] both compared an internet-based program with education only to an internetbased program with education and behavioral therapy. Both studies demonstrated significantly greater weight loss at post-intervention in the internet-based program with education and behavioral therapy. The additional behavioral therapy features across both studies were the provision of e-mail feedback from a counselor based on a participant's self- monitoring of weight, activity and eating habits, as well as a behavioral weight loss lesson. Pullen et al. [31] showed the feasibility and efficacy of an 3-month internet-delivered weight-loss intervention for a sample of rural women aged 50-69. Authors demonstrated a mean weight loss among web-site only participants of $-5.28 \mathrm{lbs}$, compared to a mean weight loss of -11.08 lbs among web-site plus peer support group. Participants of both groups received an orientation to the web-site individually but, in addition, the peer support group participated weekly in an internet asynchronous discussion group that was designed to support the women in their behavior change attempts.

A high-sized effect was found also in Bennett et al.'s study [27]. In this trial the short-term efficacy of a 3-month web-based intervention for weight loss was evaluated in comparison with a standard care condition (e.g. dietary restructuring, meal replacements, caloric intake prescriptions). Participants were primary care patients with obesity and hypertension. In the web-based intervention, patients had access to a comprehensive weight loss website for 3 months, along with four (two in-person and two telephonic) counseling sessions with a health coach. The online program ("Step Up, Trim Down") used a weight loss strategy (iOTA; interactive weight loss approach) that provides participants with a series of tailored behavior change goals that are subjected to regular self-monitoring. Participants randomized to the internet-based intervention showed a mean weight loss of -2.3 $\mathrm{kg}$, compared to a mean weight gain of $0.28 \mathrm{~kg}$ among usual care group. This trial was not included in Neve et al.'s review (2009).

Another study not included in the previous review was conducted by Digenio et al. [32], who examined the effect of 5 methods of delivering a 6-month lifestyle modification program in combination with the weight-loss medication sibutramine. In this study, the internet was used only for delivering e-mail messages. The lifestyle program involved the behavioral strategies and the support materials adapted from the Diabetes Prevention Program (www.bsc.gwu.edu/dpp/ manuals.htmlvdoc). Digenio et al. (2009) hypothesized that high-frequency face-to-face meetings with a dietitian would produce the greatest weight loss in a sample of mostly women obese, whereas self-help would be associated with the least. Weight loss with telephone and e-mail interventions were expected to fall between these 2 groups. At the end of treatment, the mean weight loss, relative to baseline, in the High frequency face-to-face and high frequency telephone-based groups was similar $(-8.9 \%$ and $-7.7 \%)$ and significantly greater than that in the other groups (low frequency face-to-face $-6.4 \%$; high frequency e-mail $-5.9 \%$ and self-help $-5.2 \%$ ). This showed that contact frequency is an important factor for weight-loss success and that a face-toface format is superior to e-mail, which in turn produced results similar to self-help.

Philip et al. [29] evaluated the feasibility and efficacy of a 3-month Internet-based weight-loss program for overweight men. The SHED-IT (Self-Help, Exercise and Diet using Information Technology) program was designed to appeal specifically to men and involved a free website (www.calorieking.com.au) that provides tools and information for self-monitoring, goal setting and social support through access the online bulletin board. Individualized 
Table 1. Study Characteristics, Effect Sizes and 95\% Confidence Intervals for Internet-Based Interventions for Weight Loss

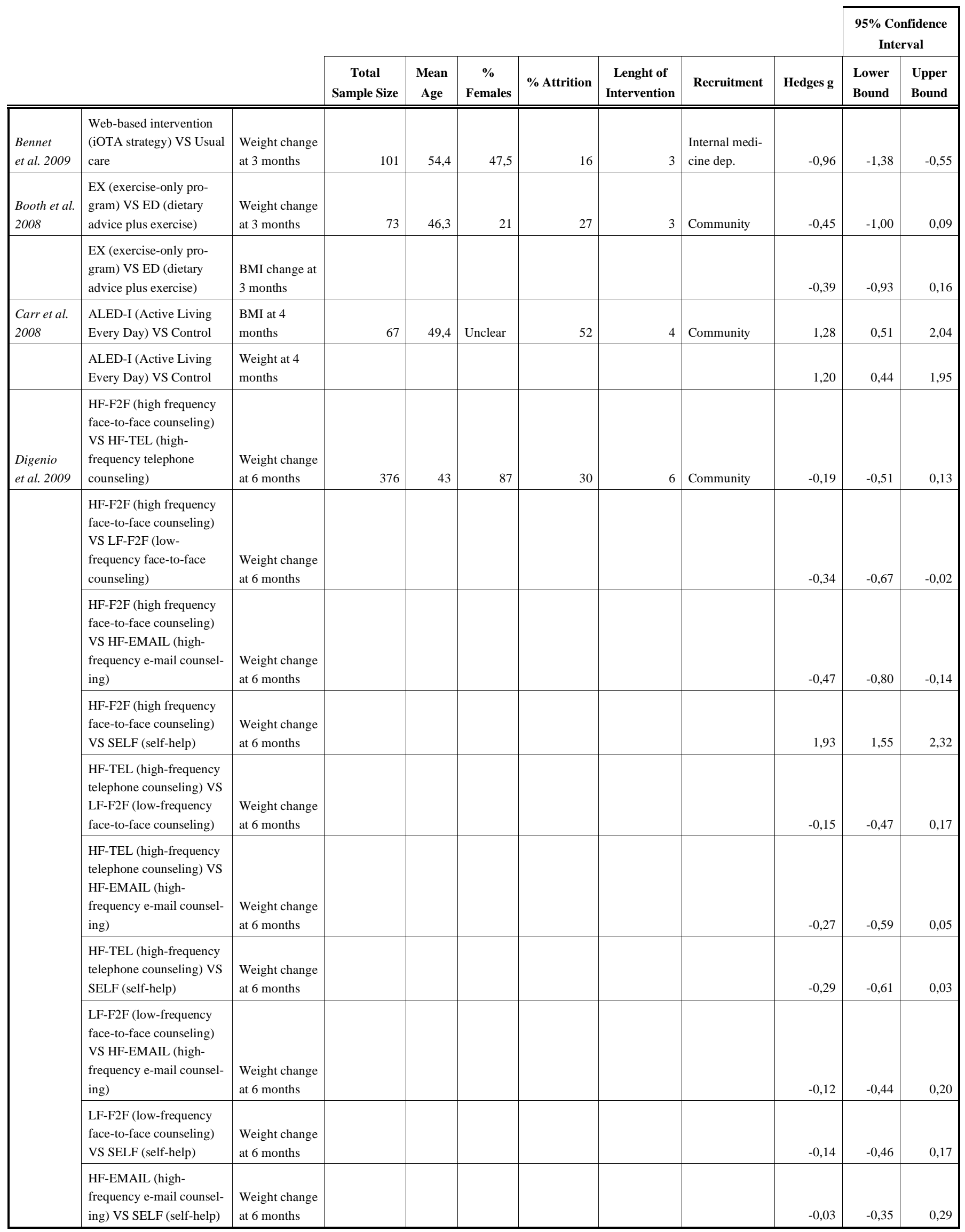


Table 1. contd....

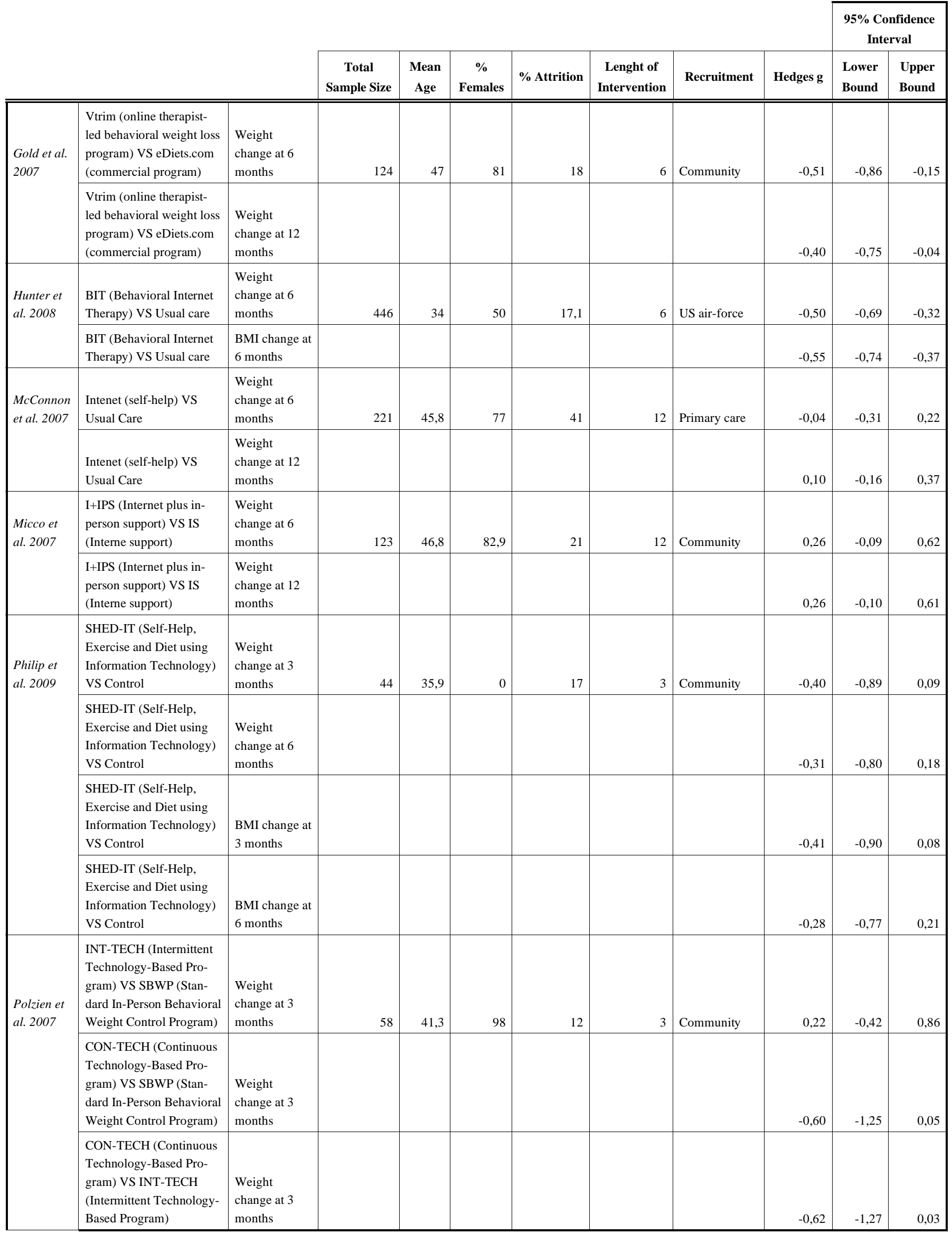


Table 1. contd....

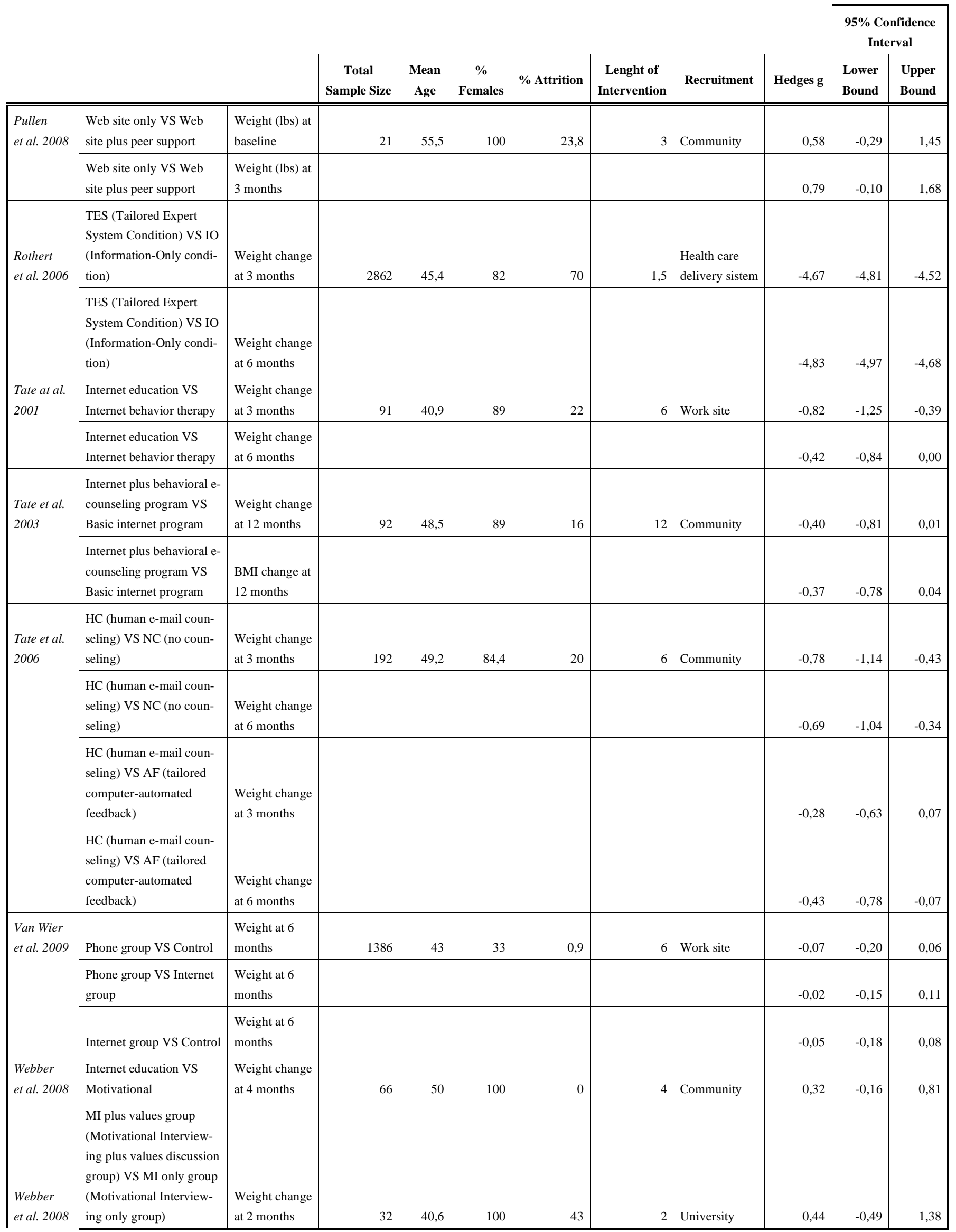


Table 1. contd....

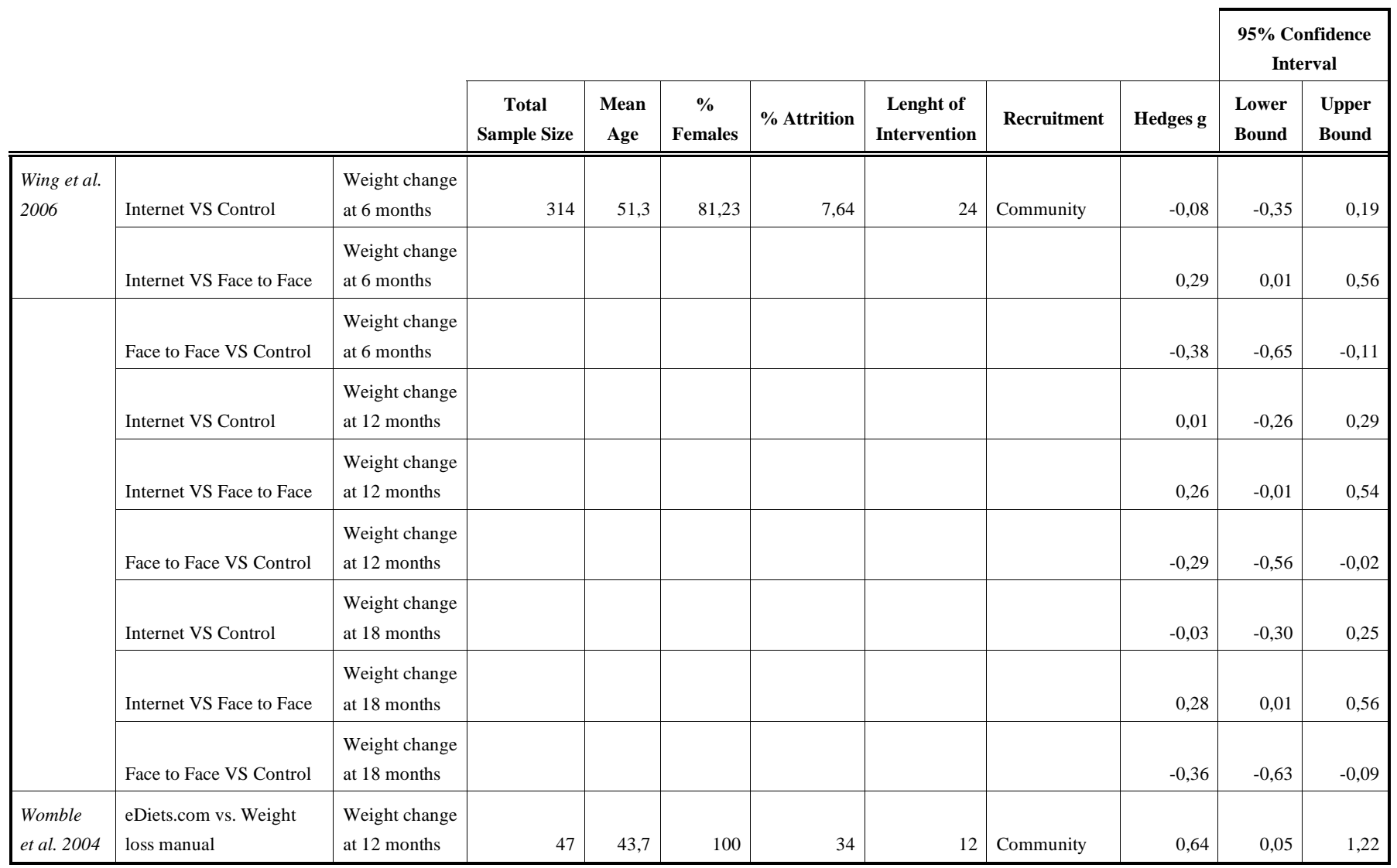

feedback corresponding to a week of diary entries were also delivered through e-mail by the research team with strategies to address weight loss, reduce energy intake and increase energy expenditure. ITT analysis revealed that both groups lost a significant amount of weight at 6-month follow-up (P $<0.001)$. However, the difference between the Internet and control groups for changes in weight from baseline to 6 months was not statistically significant $(\mathrm{p}=0.228)$.

Hunter et al. [28] evaluated the efficacy of an Internetbased program for weight-loss and weight-gain prevention in a sample of U.S. Air Force overweight men and women. All participants received usual care consisting of fitness center, weight loss and healthy cooking classes, available nutrition consultants and opportunities for individual fitness assessments and recommendations. In addition to usual care, a randomized group of participants attended an Internet-based program that included online self-monitoring, weekly personalized feedback on the food, exercise and weight information submitted, weekly lessons on the website about common strategies associated with behavioral weight loss and brief motivational interviewing telephone calls. After 6 months, participants who received the internet-based program in addition to usual care lost $1,3 \mathrm{~kg}$ while those assigned to only usual care gained $0.6 \mathrm{~kg}$. This difference was statistically significant.

Van Wier et al. [30] investigated the effectiveness of lifestyle counseling by phone or e-mail on body weight in an overweight working sample. They conducted a randomized controlled trial with three treatment arms: intervention materials with phone counseling, a web-based intervention with e-mail counseling and usual care (lifestyle brochures). All groups received self-help materials intended for the general public and dealt with overweight, healthy diet and physical activity. Additionally, the phone and internet groups received a lifestyle intervention program based on principles of behaviour therapy $[14,38]$. In particular, the internet group had access to an interactive website with individualized web pages and received feedback by a counselor through e-mail. After 6 months, both the phone group and the internet group lost significantly more weight $(-1,5 \mathrm{~kg}$ and $-0,6 \mathrm{~kg}$ respectively) than the control group, but the difference between them was not statistically significant.

Booth et al. [33] reported a twice greater weight loss percentage, but not statistically significant, in the exercise-only program group compared with the dietary advice plus exercise group in a 12-weeks online reduction program. Indeed, exercise-only participants lost $1.9 \mathrm{~kg}$ weight $(\mathrm{p}<0.01)$ and the dietary advice plus exercise group lost $0.7 \mathrm{~kg}(\mathrm{p}=0.09)$. The exercise-only program included information on exercise and monitoring of daily steps measured through a pedometer and logged on a website. Instead, dietary advice plus exercise program included an additional series of online dietary questions assessing the dietary intake of participants.

In summary, updated evidence confirm previous findings [25] that behavioral internet-based weight-loss interventions enhanced by professional feedback provided through internet is more effective that a web-site only program. However, when the internet-based counseling is compared with phone counseling, results are mixed [30,32]. New evidence that fill 
in an important demographic gap come from the Philip et al.'s study, which evaluated the effectiveness of a 3-month Internet-based weight-loss program for overweight men in comparison with a minimal intervention [29]. Data showed no statistical difference between the two groups and this result supports the evidence that internet-based interventions achieve similar weight loss to minimal intervention groups [25].

\section{Results of Studies Aiming to Achieve Weight Loss Main- tenance}

Effect sizes and relative 95\% confidence intervals for all the studies that examined the effectiveness of internet-based weight-loss maintenance program are reported in Table $\mathbf{2}$.

Apart from Cussler et al.'s study [39], which showed no further reduction in weight after completion of a maintenance program following a weight-loss intervention and no significant difference between the groups, all the other studies showed a further improvement in mean weight loss for both groups. Inspection of effect sizes and $95 \%$ confidence intervals show mixed results. In summary, as already re- marked by Neve et al. [25], evidence suggest that internetbased interventions achieve similar levels of weight loss maintenance to face-to-face interventions, and less weight is regained in comparison with no intervention. We refer the reader to Neve et al.'s study for a description of these studies.

Here we describe the Wing et al.'s study [11], the only one not included in Neve et al.'s review [25]. Wing et al. [11] randomly assigned participants who had lost a mean of $19.3 \mathrm{~kg}$ of body weight in the previous two years to one of three maintenance groups: control group, which received quarterly newsletters, a group that received face to-face intervention and a group that received Internet-based intervention. At 18-month follow-up authors found that the mean weight gain was $2.5 \mathrm{~kg}$ in the face-to-face group, $4.7 \mathrm{~kg}$ in the Internet group and $4.9 \mathrm{~kg}$ in the control group, with the face-to-face group overcoming significantly both the control group and the internet group.

\section{DISCUSSION}

This systematic review update the evidence on the effectiveness of internet-based interventions for weight loss and

Table 2. Study Characteristics, Effect Sizes and 95\% Confidence Intervals for Internet-based Intervention for Weight Loss Maintenance

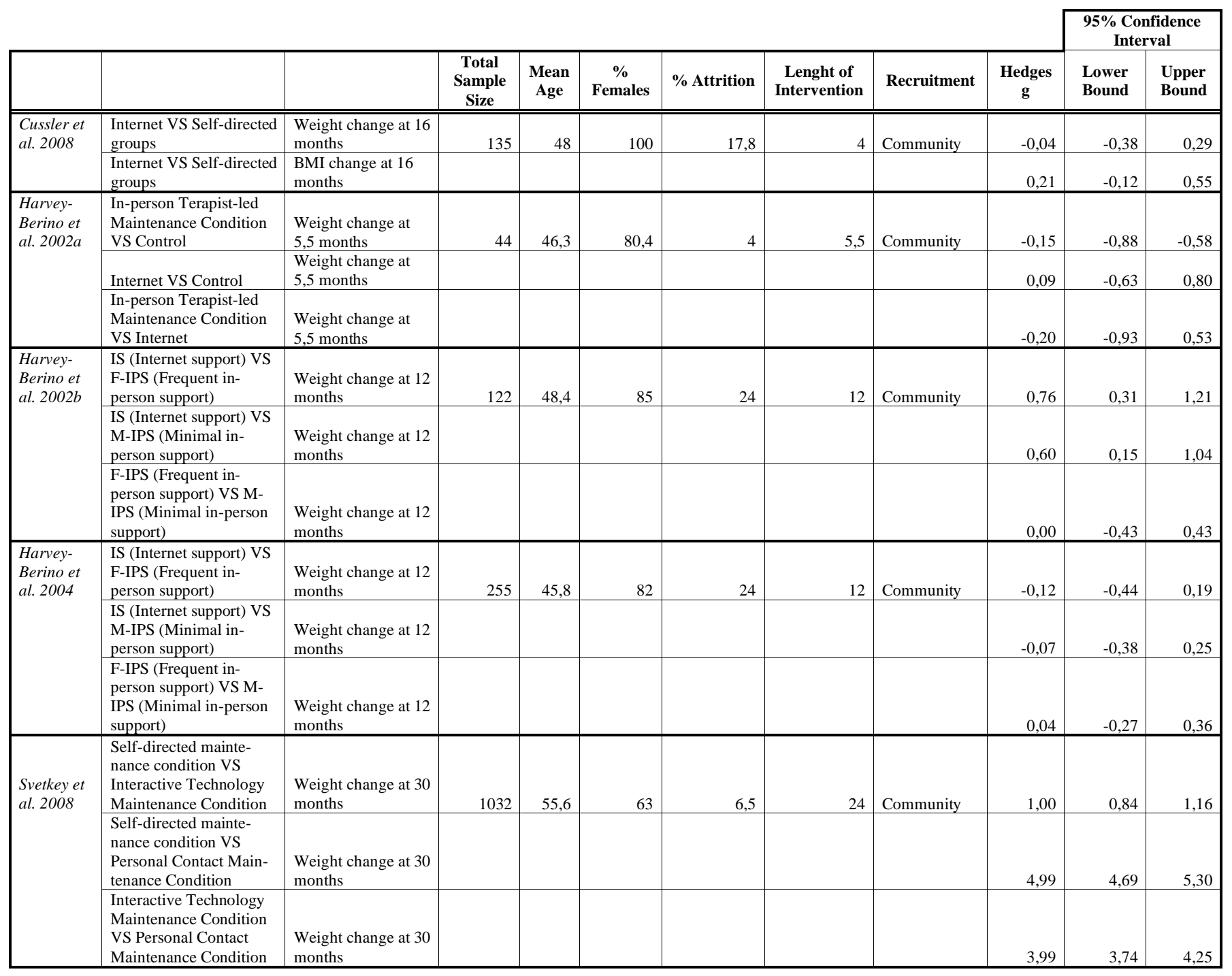


maintenance. An additional eight studies are included here, which were not part of the previous review by Neve et al. [25] that included eighteen studies. Seven of the additional studies examined internet-based interventions for weight loss [27-33], while only one evaluated a weight loss maintenance program [11]. The new evidence gained with these additional studies is modest. Philip et al.'s study provided insight into the effectiveness of an internet-based weight-loss intervention for overweight men and suggested guidelines for the development of tailored interventions for men [29]. Pullen et al.'s study provided further support to the evidence that behavioral internet-based program for weight loss enhanced by professional feedback and counseling is more effective than an education only web-site program. However, heterogeneity in designs remains a problem and precludes any conclusive statement.

Reasons for such uncertainty are manifold. First of all, studies that have been conducted up to date on this topic are very heterogeneous and any attempt to compare the results must be viewed with caution. Indeed, internet-based programs used in these trials differ from each other on many features (length of intervention, type and frequency of feedback, use of social support tools, etc.) and are also compared with control or comparison conditions that vary a lot among studies. Furthermore, no study has compared an internetbased program with a "real" control group. Although this heterogeneity among studies in interventions and controls may be informative at study level, it makes meta-analysis an untenable or impossible task. Indeed, among the previous reviews, one only attempted to synthesize data with a metaanalytical method [25], but the overall effect sizes that were calculated by pooling two or three similar studies are far from being reliable.

Secondly, no study has directly compared an internetbased intervention with a traditional individual or groupbased behavioral treatment. Comparing mean weight loss obtained in the study with data from classical behavioral trials examining face-to-face interventions may be informative, but this approach is biased due to differences in samples and cannot replace a randomized study specifically designed to test a difference between the two formats of delivering a weight-loss behavioral treatment.

Thirdly, although internet has the potential to reduce costs of treatment delivery, only two studies out of the 26 reviewed in this paper assessed cost-effectiveness of an internet-based intervention [33, 40]. According to Booth et al. [33], while the initial construction of the web site itself is relatively costly, ongoing costs are minimal. The authors found that, based on the minimum cost of consulting a dietitian in a public hospital, an initial 1-hour consultation (\$70) plus fortnightly 30-min follow-up consultations (\$35) would total $\$ 280$ over a period of 12 weeks. Instead, a 0.5 -hour initial consultation $(\$ 35)$, followed by plus $15 \mathrm{~min}$ at week (\$30 per hour) input from a nutritionist using an Internetbased program would total only $\$ 125$ for the 12 -week program. Additional costs would be saved for participants through elimination of travel costs and travel time, with Internet costs being minimal for those already connected [33].

Four, no study examined gender differences and generalization of results to men is questionable. Indeed, men are very under-represented in the studies on internet-based weight loss programs, except for one that recruited only men [29], and the moderator effect of gender on weight outcomes or treatment adherence is unknown.

Five, although most studies have shown a significant association between higher compliance to treatment demands and greater weight loss and weight loss maintenance [25], a standard method to evaluate adherence was not used and no meta-regression can thus be performed to examine that association in a meta-analytical fashion. Most studies reported findings as an average number of contacts with a particular feature, therefore not allowing comparability between them. According to Neve et al. [25], future controlled randomized trials should report compliance as a proportion of recorded to expected contacts with the particular web-feature.

Furthermore, future studies should adopt one of the methods to account for noncompliance [41]. Among the studies included in the present review, one only used such a method, i.e. per-protocol analysis [29].

Six, given the complex structures of the weight-loss programs delivered in the reviewed studies, many unanswered questions concern the components, the mechanisms and the mediators that lead to weight loss and maintenance. At present, the only factor that has received some empirical support is e-mail contact with a professional counselor that provides feedback and reinforcement along the intervention. However, although Internet programs with counseling from a human therapist may make treatment more effective than automated e-counseling [37], developing technologies make virtual counselors possible [42]. Other models proposed by Winett et al. [42] may combine virtual counselors or tailored messages with intermittent use of human counselors to make more disseminable lower cost treatments.

Finally, most of the studies reviewed in this paper recruited overweight or obese participants from the community. None of them have been done with obese patients referred to specialty clinics or to bariatric surgery.

\section{CONCLUSION}

The Internet offers a novel delivery tool for weight loss and weight loss maintenance interventions, with the potential to offer long-term intervention at a low cost, in comparison to traditional face-to-face treatments. However, the evidence of effectiveness is modest due to mixed results, heterogeneity of designs and low generalization of findings. Future research in the area should prioritize well-designed efficacy trials that account for known sources of variation and determine which features of internet-based interventions are critical to achieve success in weight loss and maintenance in a cost-effective and time-saving manner. Future research should also develop and evaluate internet-based weight-loss interventions that are specifically tailored to the needs of the health-care delivery system.

\section{REFERENCES}

[1] WHO. Fact sheet $\mathrm{N}^{\circ} 311$ Obesity and overweight. Available from: http://www.who.int/mediacentre/factsheets/fs311/en/index.html.

[2] Whitlock G, Lewington S, Sherliker P, et al. Body-mass index and cause-specific mortality in 900000 adults: collaborative analyses of 57 prospective studies. Lancet 2009; 373: 1083.

[3] Flegal KM, Graubard BI, Williamson DF, Gail MH. Excess deaths associated with underweight, overweight, and obesity. JAMA 2005; 293: 1861 . 
[4] Dixon JB. The effect of obesity on health outcomes. Mol Cell Endocrinol 2009; 316: 104.

[5] Pi-Sunyer FX. Clinical guidelines on the identification, evaluation, and treatment of overweight and obesity in adults--the evidence report. national institutes of health. Obes Res 1998; 6: 51S.

[6] Levy RL, Finch EA, Crowell MD, Talley NJ, Jeffery RW. Behavioral intervention for the treatment of obesity: strategies and effectiveness data. Am J Gastroenterol 2007; 102: 2314.

[7] Wadden TA, Butryn ML, Byrne KJ. Efficacy of lifestyle modification for long-term weight control. Obes Res 2004; 12 Suppl: 151S.

[8] Weinstein PK. A review of weight loss programs delivered via the Internet. J Cardiovasc Nurs 2006; 21: 251.

[9] Perri MG, Sears SF, Jr, Clark JE. Strategies for improving maintenance of weight loss. Toward a continuous care model of obesity management. Diabetes Care 1993; 16: 200.

[10] Hill JO, Thompson $\mathrm{H}$, Wyatt $\mathrm{H}$. Weight maintenance: what's missing? J Am Diet Assoc 2005; 105: S63.

[11] Wing RR, Tate DF, Gorin AA, Raynor HA, Fava JL. A selfregulation program for maintenance of weight loss. N Engl J Med 2006; 355: 1563.

[12] Jeffery RW, Drewnowski A, Epstein LH, et al. Long-term maintenance of weight loss: current status. Health Psychol 2000; 19: 5.

[13] Katan MB. Weight-loss diets for the prevention and treatment of obesity. N Engl J Med 2009; 360: 923.

[14] Wadden TA, Crerand CE, Brock J. Behavioral treatment of obesity. Psychiatr Clin North Am 2005; 28: 151.

[15] Polzien KM, Jakicic JM, Tate DF, Otto AD. The efficacy of a technology-based system in a short-term behavioral weight loss intervention. Obesity (Silver Spring) 2007; 15: 825.

[16] Sherwood NE, Morton N, Jeffery RW, French SA, NeumarkSztainer D, Falkner NH. Consumer preferences in format and type of community-based weight control programs. Am J Health Promot 1998; 13: 12 .

[17] Tate DF, Wing RR, Winett RA. Using Internet technology to deliver a behavioral weight loss program. JAMA 2001; 285: 1172 .

[18] Hellerstedt WL, Jeffery RW. The effects of a telephone-based intervention on weight loss. Am J Health Promot 1997; 11: 177.

[19] Meyers AW, Graves TJ, Whelan JP, Barclay DR. An evaluation of a television-delivered behavioral weight loss program: are the ratings acceptable? J Consult Clin Psychol 1996; 64: 172.

[20] Harvey-Berino J. Changing health behavior via telecommunications technology: using interactive television to treat obesity. Behav Ther 1998; 29: 505.

[21] Taylor CB, Agras WS, Losch M, Plante TG, Burnett K. Improving the effectiveness of computer-assisted weight loss. Behav Ther 1991; 22: 229.

[22] Burnett KF, Taylor CB, Agras WS. Ambulatory computer-assisted therapy for obesity: A new frontier for behavior therapy. J Consult Clin Psychol 1985; 53: 698.

[23] Tsai AG, Wadden TA. Systematic review: an evaluation of major commercial weight loss programs in the United States. Ann Intern Med 2005; 142: 56.

[24] Tsai AG, Wadden TA, Womble LG, Byrne KJ. Commercial and self-help programs for weight control. Psychiatr Clin North Am 2005; 28: 171

[25] Neve M, Morgan PJ, Jones PR, Collins CE. Effectiveness of webbased interventions in achieving weight loss and weight loss main- tenance in overweight and obese adults: a systematic review with meta-analysis. Obes Rev 2009; 11(4): 306-21.

[26] Saperstein SL, Atkinson NL, Gold RS. The impact of Internet use for weight loss. Obes Rev 2007; 8: 459.

[27] Bennett GG, Herring SJ, Puleo E, Stein EK, Emmons KM, Gillman MW. Web-based weight loss in primary care: a randomized controlled trial. Obesity (Silver Spring) 2009; 18: 308.

[28] Hunter CM, Peterson AL, Alvarez LM, et al. Weight management using the internet a randomized controlled trial. Am J Prev Med 2008; 34: 119.

[29] Philip JM, Lubans DR, Collins CE, Warren JM, Callister R. The SHED-IT Randomized Controlled Trial: Evaluation of an Internetbased Weight-loss Program for Men. Obesity (Silver Spring) 2009; 17: 2025.

[30] van Wier MF, Ariens GA, Dekkers JC, Hendriksen IJ, Smid T, van Mechelen W. Phone and e-mail counselling are effective for weight management in an overweight working population: a randomized controlled trial. BMC Public Health 2009; 9: 6.

[31] Pullen CH, Hageman PA, Boeckner L, Walker SN, Oberdorfer MK. Feasibility of Internet-delivered weight loss interventions among rural women ages 50-69. J Geriatr Phys Ther 2008; 31: 105.

[32] Digenio AG, Mancuso JP, Gerber RA, Dvorak RV. Comparison of methods for delivering a lifestyle modification program for obese patients: a randomized trial. Ann Intern Med 2009; 150: 255.

[33] Booth AO, Nowson CA, Matters H. Evaluation of an interactive, Internet-based weight loss program: a pilot study. Health Educ Res 2008; 23: 371.

[34] Rothert K, Strecher VJ, Doyle LA, et al. Web-based weight management programs in an integrated health care setting: a randomized, controlled trial. Obesity (Silver Spring) 2006; 14: 266.

[35] Gold BC, Burke S, Pintauro S, Buzzell P, Harvey-Berino J. Weight loss on the web: A pilot study comparing a structured behavioral intervention to a commercial program. Obesity (Silver Spring) 2007; $15: 155$.

[36] Tate DF, Jackvony EH, Wing RR. Effects of Internet behavioral counseling on weight loss in adults at risk for type 2 diabetes: a randomized trial. JAMA 2003; 289: 1833.

[37] Tate DF, Jackvony EH, Wing RR. A randomized trial comparing human e-mail counseling, computer-automated tailored counseling, and no counseling in an Internet weight loss program. Arch Intern Med 2006; 166: 1620.

[38] Fabricatore AN. Behavior therapy and cognitive-behavioral therapy of obesity: is there a difference? J Am Diet Assoc 2007; 107: 92.

[39] Cussler EC, Teixeira PJ, Going SB, et al. Maintenance of weight loss in overweight middle-aged women through the Internet. Obesity (Silver Spring) 2008; 16: 1052.

[40] McConnon A, Kirk SF, Cockroft JE, et al. The Internet for weight control in an obese sample: results of a randomised controlled trial. BMC Health Serv Res 2007; 7: 206.

[41] Hertogh EM, Schuit AJ, Peeters PH, Monninkhof EM. Noncompliance in lifestyle intervention studies: the instrumental variable method provides insight into the bias. J Clin Epidemiol 2010; 63(8): 900-6.

[42] Winett RA, Tate DF, Anderson ES, Wojcik JR, Winett SG. Longterm weight gain prevention: a theoretically based Internet approach. Prev Med 2005; 41: 629. 